\title{
Una evaluación geográfica de la política de educación media Superior de la Ciudad de México
}

\author{
Laura Cervantes-Salcido* \\ Carlos J. Vilalta-y Perdomo**
}

\section{Resumen}

En este trabajo presentamos una evaluación geográfica de la política de las preparatorias creadas y administradas por el gobierno de la ciudad de México. La revisión teórica y evaluación estadística realizadas nos ha permitido construir relaciones explicativas útiles entre varios conceptos de teorías urbanas provenientes de escuelas divergentes, y de disciplinas diferentes, y a la vez hacer una demostración empírica del incumplimiento de uno de los objetivos geográficos de esta política de la ciudad de México.

Palabras clave: política pública, educación pública, distrito federal, geografía humana, análisis geográfico.

\section{Abstract}

In this paper we present a geographical evaluation of the policies for preparatorias (bigh schools) created and administered by the government of Mexico City. The theoretical revision and statistical evaluation that were carried out have allowed us to construct useful relationships that explain various concepts from urban theories arising from diverging schools of thought and different disciplines. At the same time, it has been possible to demonstrate empirically the nonfulfillment of one of the geographical objectives of this policy in Mexico City.

Keywords: Public policy, public education, Distrito Federal, human geography, geographic analysis

* Tecnológico de Monterrey, Campus Santa Fe, Correo-e: lcervant@itesm.mx

** Tecnológico de Monterrey, Campus Santa Fe, University of North Carolina, Chapel Hill. Correo-e: carlos.vilalta@itesm.mx, carlos.vilalta@itesm.mx 


\section{Introducción}

La educación urbana es un tema de política pública sumamente significativo y polémico, pero curiosamente pobremente documentado en México. Es inclusive uno de los temas más recurrentes en discursos oficiales y campañas electorales locales y federales. No obstante lo anterior, la educación pública no es un tema que haya sido analizado de manera cuantitativa y geográfica por los urbanistas mexicanos.

En la literatura académica mexicana se pueden encontrar unos pocos estudios recientes -no geográficos o urbanísticos- enfocados a estudiar las tendencias homogámicas en la educación de la sociedad mexicana (Esteve, 2005), la relación entre el gobierno local y las instituciones de educación superior en un contexto de alternancia política (Acosta, 2004), el rendimiento económico de la educación (Barceinas, 2003), y la brecha de género educativa existente en México (Parker y Pederzini, 2000). Esas perspectivas son diversas, y los estudios, valiosos en sí mismos en cuanto a los hallazgos y las evidencias presentados. Sin embargo, en relación con nuestros intereses más particulares, en nuestra revisión de la literatura académica en México no pudimos encontrar un estudio que analizara científicamente la localización geográfica de las escuelas públicas, ni tampoco una discusión sobre si ésta es socialmente justa y/o económicamente óptima. En síntesis, no son visibles -si es que los hay- estudios teóricoempíricos que analicen la geografía urbana o rural de las políticas públicas de educación.

Los únicos estudios cuantitativos existentes sobre educación pública son realizados por el gobierno federal y se concentran en el análisis y proyección de diversas variables clásicas como son: la deserción escolar, la retención, la eficiencia terminal y el alumnado total. ${ }^{1}$ Sin embargo, todos -sin excepción- carecen de una visión analítica-geográfica. De manera concreta, cuantitativa y geográficamente hablando, lo que usualmente se hace es contabilizar escuelas y realizar mediciones descriptivas (indicadores) en los ámbitos, estatal y municipal.

Como mencionábamos, el tema de las escuelas públicas es materia discursiva de la política en México, pero la localización geográfica de éstas también es materia central en las labores de

\footnotetext{
${ }^{1}$ La mayoría de esos estudios son realizados por la Secretaría de Educación Pública del gobierno federal de México.
} 
planeación urbana. Una perspectiva geográfica en la planeación de la educación pública es indispensable y decisiva, ya que de manera similar a los demás servicios públicos urbanos, la educación obedece a una lógica espacial conceptualmente simple: es preciso ofrecerla en el lugar donde se requiere.

En este trabajo realizamos, precisamente, una evaluación geográfica de la política de educación media superior del Distrito Federal -o ciudad de México- con base en: a) la importancia social de la educación pública, $b$ ) su propia lógica geográfica $\mathrm{y}$, relacionada con los dos puntos anteriores, $c$ ) la carencia de análisis estadístico-geográficos sobre este tipo de políticas públicas. Nos concentramos en estudiar la localización de las preparatorias administradas por el Sistema de Bachillerato del Gobierno del Distrito Federal (sBgDF).

Cabe mencionar que esta política de educación media superior local incluye en su discurso, como elemento distintivo, el criterio geográfico para la localización de los planteles $(\mathrm{n}=16)$. En específico, en ella se establece que la ubicación de las preparatorias del SBGDF estará determinada por un criterio de alta marginación. Su objetivo social ha sido el de atender la demanda de educación media superior en las zonas de mayor pobreza del Distrito Federal. También cabe adelantar que el Gobierno del Distrito Federal (GDF), de filiación perredista, ha sido un gobierno urbano que se ha caracterizado consistentemente -de manera discursiva y factual- por promover políticas de fuerte contenido social; o sea, políticas redistributivas (Peterson, 1981). El GDF ha pretendido, con esta política de educación media, identificar geográficamente la demanda de la población más necesitada y satisfacerla.

Nuestra pregunta de investigación sobre esta política redistributiva deriva precisamente del criterio geográfico descrito, y es la siguiente: ¿están los planteles del SBGDF efectivamente localizados en las zonas más marginadas de la ciudad?

Adelantamos que los resultados de este estudio muestran que el objetivo geográfico del SBGDF no se cumple. La ubicación de los planteles no corresponde con el objetivo político expreso de localización en las zonas de mayor marginación social de la ciudad. Más bien, la evidencia y las pruebas que realizamos y presentamos en este estudio muestran que los planteles han sido ubicados en zonas de la ciudad que no son las que presentan los mayores niveles de marginación social. En síntesis, esta política urbana no posee la eficacia geográfica y redistributiva que pretende. 
Para explicar los resultados y discutir al respecto, este documento se divide en las siguientes partes: teorías y conceptos, metodología y datos, resultados, y discusión y conclusiones.

\section{Teorías y conceptos: política urbana, consumo colectivo, externalidades y localización óptima}

La temática urbana es tan variada y parece estar tan fragmentada que para algunos autores es debatible si la ciudad es una entidad coherente para la aplicación de las ciencias sociales o si es más concretamente un "objeto social unitario" (Abrams, 1978: 15). Nosotros defendemos la posición de que independientemente de esta complejidad temática y conceptual, no hay duda de que la ciudad tiene un valor epistemológico propio (Handlin, 1967) y existen perspectivas teóricas sociales y formas científicas probadas para definir, analizar e interpretar lo urbano. Pensamos, como lo hacen la mayor parte de los sociólogos urbanos en México, que los fenómenos sociales urbanos son clasificables y asociables a regularidades empíricas y que por lo tanto son susceptibles de entenderse y teorizarse.

Además de lo anterior, las diversas teorías sociales urbanas tienen una perspectiva geográfica implícita en sus formulaciones (Vilalta, 2003). Es decir, en algún punto estas teorías entrecruzan la dimensión espacial con las demás ciencias sociales para atribuirle un sentido a los fenómenos urbanos. En consecuencia, estas teorías tratan temas y ofrecen clasificaciones y explicaciones muy variadas, pero clara y necesariamente interrelacionadas; los fenómenos urbanos son por naturaleza transdisciplinarios.

Debido a la cualidad de transdisciplinario de lo urbano, en este estudio utilizamos dos perspectivas teóricas clásicas -marxismo y economía clásica- que nos permiten precisamente realizar la conexión entre cuatro diferentes temas sociales también clásicos en el análisis urbano. Esos cuatro temas están directamente relacionados con el asunto principal de nuestro estudio: la lógica geográfica de la educación pública. El beneficio evidente de utilizar varias bases teóricas y con ellas procurar la conexión entre esos temas y conceptos analíticos urbanos, es que nos ayuda a observar y entender mejor la lógica geográfica que existe detrás de la provisión de los servicios públicos en las áreas urbanas; nuevamente, la lógica geográfica de la educación pública. 
Analítica y detalladamente, los conceptos interrelacionados que revisamos a continuación son: $a$ ) la política urbana, $b$ ) el consumo colectivo de los servicios públicos, $c$ ) las externalidades económicas, y $d$ ) la localización óptima. Al final de la sección logramos mostrar dos cosas: que para efectos teóricos, la combinación de estos conceptos resulta sumamente útil, rica y nítida, pero que en ocasiones la relación entre ellos no es perfecta cuando llegamos a su aplicación real en la planeación urbana. Al contrario, la correcta y simultánea puesta en práctica de esos conceptos es muy difícil de realizar.

Entonces, en detalle, utilizamos primeramente una perspectiva teórica clásica de fundamento marxista para explicar la compleja pero consustancial relación entre política urbana, consumo colectivo y externalidades económicas. Posteriormente, utilizamos una perspectiva económica clásica aplicada a la geografía urbana para explicar con más especificidad la relación entre consumo colectivo y localización óptima. Finalmente, en esta sección, y a fin de contextualizar, presentamos un breve resumen de las características y objetivos geográficos de la política de las preparatorias planeadas y administradas por el SBGDF.

\subsection{Política urbana, consumo colectivo y externalidades económicas}

Históricamente, los gobiernos urbanos han sido los encargados de la provisión de los servicios elementales para el sostenimiento social y económico de las ciudades; servicios tales como el drenaje, la transportación, el agua, la electricidad, la recreación, etcétera. $^{2}$

Una de las condiciones generales de la producción (Garza y Sobrino, 1989) es el servicio público de la educación, la cual está naturalmente ligada al ciclo del capital y de la reproducción de la fuerza de trabajo en la ciudad, y a un consumo colectivo de la misma. Desde de la perspectiva teórica marxista, la educación es un servicio de consumo colectivo desde el momento en que forma parte elemental del proceso de reproducción del capital y del trabajo, y cuando el Estado interviene en su organización para el

\footnotetext{
${ }^{2}$ El grado de acceso a los bienes y servicios constituye un aspecto fundamental y un indicador del bienestar social. Generalmente, uno de los rasgos notablemente penosos en los países en desarrollo, es la diferencia entre la gente pobre y rica, condición que incluye importantes divergencias en el acceso a servicios.
} 
consumo generalizado. Recordemos que en esta visión marxista, el papel del Estado es asegurar la reproducción de la estructura social y garantizar la supervivencia del capital (Castells, 1974).

Como las demás áreas de intervención del Estado, la educación es un área de planeación logística y políticamente compleja. La complejidad logística deriva de carencias presupuestales y deficiencias administrativas, a veces aunadas a un rápido cambio demográfico. La complejidad política deriva de la variedad en el tipo y el número de agentes políticos que demandan este servicio y que participan en el proceso político de su consumo. Los agentes involucrados se pueden encontrar en todos los sectores de la sociedad: el sector privado, el sector público autónomo, organizaciones comunitarias (movimientos urbanos populares) y los mismos gobiernos federales y estatales.

En años recientes, la respuesta gubernamental mexicana al auge democrático, por un lado, y a las dificultades económicas, por el otro, ha sido de tipo neoliberal, lo que ha implicado la retirada relativamente rápida del gobierno en el control y regulación de algunos aspectos en el mercado de bienes y de servicios en general. Sin embargo, la política en educación en México no ha sido mayormente afectada o modificada por esta corriente de reformas neoliberales. El gobierno mexicano, en los niveles federal y estatal, sigue involucrado en la planeación y provisión de la educación, y retiene sus facultades legales en cuanto a la definición de estándares y requisitos de calidad sobre el sector privado de la educación. Lo que sí ha sucedido es un incremento muy importante en el número de instituciones privadas, en todos los niveles, que han venido a cubrir la demanda creciente de educación.

Desde el punto de vista de teoría económica urbana, la intervención de un gobierno local en la provisión de un servicio está plenamente justificada cuando ese servicio se ofrece en condiciones que pueden derivar en un monopolio natural, o bien, y de manera muy importante, cuando esa misma provisión del servicio puede generar externalidades positivas (Sullivan, 1990); tal es precisamente el caso de la educación.

Los efectos negativos de la provisión de servicios urbanos en condiciones monopólicas ya han sido discutidos de manera extensiva y acertada en otros trabajos (Henderson y Ledebur, 1972; Hirsch, 1973; Sullivan, 1990). En cambio, la relación entre el consumo colectivo de un servicio y las externalidades económicas - positivas o negativas- no se ha tratado con mucha frecuencia o detalle (Stahl y Varaiya, 1983; Sandler, 1992). 
Una externalidad económica ocurre cuando el consumo de un bien o servicio por una persona genera beneficios o costos para otras personas. Estas externalidades tienen efectos diferenciales en esos beneficios y costos, tienen efectos privados y sociales. Un efecto clásico es que los consumidores del servicio, quienes basan su decisión de consumo en su propio beneficio y costo, toman decisiones que pueden ser ineficientes para el conjunto de las demás personas (Potters y Van Winden, 1996). Es precisamente por estos efectos diferenciales que se argumenta que la intervención del gobierno en la producción de ciertos bienes o servicios puede traducirse, simultáneamente, en un consumo colectivo más justo -una visión marxista- y en una promoción más eficiente en el mercado -una visión de economía clásica.

Dentro de la lógica de una economía urbana, Sullivan (1990) menciona que la educación produce justamente un tipo de externalidad positiva recíproca al generar beneficios privados tanto para el consumidor -el estudiante- como para la sociedad. El beneficio social puede igualmente verse desde un matiz marxista (Castells, 1974) considerando al estudiante como un futuro trabajador cualificado y rentable. En general, la lógica marxista de la provisión pública de la educación es que el efecto final resultante será que cuanto mejor preparados estén los trabajadores, mayores serán sus capacidades y su rentabilidad; "adicionalmente, con ciudadanos más educados se toman mejores decisiones, puesto que el beneficio social marginal de la educación excede el beneficio privado marginal" (Sullivan, 1990: 421).

Finalmente, cabe decir que los gobiernos urbanos tienen dos opciones para generar estas externalidades positivas. Por un lado, pueden tomar la responsabilidad de proveer directamente dicho servicio educativo con la creación y mantenimiento de escuelas gratuitas. Por otro lado, pueden otorgar subsidios para que cada estudiante elija dónde estudiar, como sucede en algunos estados de Estados Unidos. En México, a la fecha se ha decidido seguir con la primera opción.

\subsection{Consumo colectivo y localización óptima}

Todos los servicios públicos poseen una zona específica de oferta dentro de la cual los consumidores se trasladan de su lugar de residencia para el uso del servicio (Werna, 2001). Teóricamente, estas zonas de oferta tienen dos límites geográficos: un límite mínimo, que se define por el área dentro de la cual se asegura la 
viabilidad logística y económica del servicio, y un límite máximo, que es precisamente el máximo alcance hasta el cual el consumidor se desplazaría para adquirir el servicio (Werna, 2001).

Relacionado con lo anterior, Bola (1979) supuso ya hace tiempo que el suministro de servicios públicos en áreas urbanas requiere considerar el grado de accesibilidad y la carga en tiempo y costos de traslado que los gobiernos urbanos imponen a los usuarios de esos servicios. Recordemos que en economía urbana y regional, la localización óptima es función del grado de accesibilidad.

La accesibilidad determina que el servicio se considere óptimamente localizado cuando da -al lugar donde se ofrece el serviciouna ventaja comparativa sobre otros lugares en cuanto a la reducción de la fricción de la distancia (Bola, 1979). En otras palabras, la accesibilidad y la localización se refieren a la utilidad y conveniencia que la ubicación de un servicio puede representar para sus consumidores objetivo. Sólo hay un consumo colectivo justo y eficaz cuando se cuenta con una localización adecuada.

Naturalmente, el consumo colectivo justo y eficaz de la educación pública es dependiente de un equilibrio geográfico entre demanda y oferta. Por lo tanto, para efectos de planeación urbana, la ubicación de un plantel en una parte de la ciudad implica necesariamente la existencia de un número mínimo, suficiente, de usuarios; en este caso, estudiantes dentro del rango de edades escolares a los que se pretende atender. También para efectos de planeación, la medición de la accesibilidad y localización puede ser realizada en términos de distancia, por tiempo de traslado invertido, por costo monetario, o por una combinación de los indicadores anteriores.

El asunto crucial en la localización de un servicio de consumo colectivo es considerar su centralidad para facilitar la provisión a la mayor cantidad de usuarios objetivo de la política de ese servicio. Es decir, la educación publica debe ofrecerse lo más cerca posible a las áreas con la mayor necesidad y maximizarse con ello sus externalidades positivas.

\subsection{Las preparatorias del Sistema de Bachillerato del Gobierno del Distrito Federal}

Uno de los factores principales que ha orientado el desarrollo del SBGDF es expresamente geográfico: poner en marcha "planteles estratégicamente distribuidos en el territorio del Distrito Federal” (sBGDF, 2002: 7). Por lo mismo, la política de localización 
de las preparatorias de la ciudad de México tenía el propósito original de hacerlas geográficamente accesibles para el consumo colectivo de los grupos más marginados de la ciudad. Esto es, se decidió ubicarlas en las zonas más pobres de la ciudad, en cada delegación, y para el uso de los estudiantes de esas mismas zonas urbanas marginadas.

La primera preparatoria del SBGDF fue inaugurada en 1998 en la delegación Iztapalapa. ${ }^{3}$ Esta preparatoria fue resultado de la articulación exitosa de dos agentes políticos diferentes (el GDF y distintas organizaciones sociales de Iztapalapa), pero con el mismo objetivo: el interés por fortalecer la educación pública y gratuita en la ciudad.

Para el año 2001, los objetivos del SBGDF se volvieron más ambiciosos, y como resultado se amplió el número de planteles hasta completar un total de 16. Esta ampliación pretendió igualmente seguir el criterio de localizar los planteles en las zonas de mayor marginación; más técnicamente, en las zonas con los mayores índices de marginación de cada una de las 16 delegaciones en que está dividida la ciudad para su administración.

Está establecido también que en cada uno de los planteles del sistema solamente se puede dar cabida a 1,050 alumnos. Entre los requisitos de inscripción, los estudiantes tienen que realizar un examen de admisión y tener su domicilio a no más de tres kilómetros del plantel de su interés.

Por lo tanto, esta política contiene dos objetivos geográficos explícitos:

- La localización de los planteles en las zonas de mayor marginación de la ciudad.

- La admisión es exclusiva para los alumnos residentes de esas zonas marginadas, definiéndose la residencia en un radio de tres kilómetros a la redonda del plantel.

En este trabajo nos abocamos estrictamente a probar si la política cumple con el primer objetivo, es decir, el geográfico. Al respecto, y como adelantamos en la Introducción, en este estudio encontramos que la política no cumple con dicha meta, puesto que los planteles no están localizados en las zonas de mayor marginación de la ciudad.

\footnotetext{
${ }^{3}$ Esta preparatoria se ubicó en las instalaciones de la ex-cárcel de mujeres, en Santa Martha Acatitla.
} 


\section{Metodología y datos}

En este trabajo nos concentramos en la prueba del primer objetivo territorial de la política de educación media de la ciudad de México instrumentada por el SBGDF.

Estadísticamente, nuestra hipótesis de investigación es la siguiente:

- Ho: No hay una diferencia significativa en los niveles de marginación entre las zonas donde hay preparatorias localizadas y las zonas donde no las hay.

- Ha: Sí hay una diferencia significativa en los niveles de marginación entre las zonas donde hay preparatorias localizadas y las zonas donde no las hay.

Para no rechazar la hipótesis nula, sería requisito que los niveles de marginación socioeconómica fueran similares en los dos tipos de zonas: en las zonas que están cubiertas por las preparatorias (ZC) y en las zonas que no lo están (ZNC).

Puede suceder, en cambio -y sucede de hecho-, que los niveles de marginación sean más altos en las zonas donde no hay preparatorias localizadas versus las zonas en donde sí fueron localizadas. En síntesis, para argumentar que la localización ha sido la correcta de acuerdo con el objetivo de mayor marginación, es requisito indispensable que el promedio de los niveles de marginación sea significativamente más alto en las zonas donde se ha decidido localizar las preparatorias (zc) frente a las otras zonas

\section{Cuadro 1}

Técnica para la prueba de hipótesis, posibles resultados y conclusión

\begin{tabular}{lcc}
\hline Técnica & Posibles resultados & Conclusión \\
\hline Mann-Whitney & $\mu_{\mathrm{zc}}>\mu_{\mathrm{znc}}$ & Localización correcta \\
& $\mu_{\mathrm{zc}}=\mu_{\mathrm{znc}}$ & Localización correcta \\
$\mu_{\mathrm{zc}}<\mu_{\mathrm{znc}}$ & Localización incorrecta \\
\hline Donde: & \\
$\mu_{\mathrm{zc}}:$ Promedio del Índice de Marginación en las zonas cubiertas (zc) por \\
preparatorias \\
$\mu_{\mathrm{znc}}: \begin{array}{l}\text { Promedio del Índice de Marginación en las zonas no cubiertas (zNc) por } \\
\text { preparatorias }\end{array}$ \\
\hline
\end{tabular}

Fuente: Elaboración propia. 
que han sido excluidas de la política (ZNC), o, por lo menos, que no sean diferentes (véase cuadro 1).

Para poder distinguir metodológicamente entre las zonas que cuentan con una preparatoria del GDF, es decir, aquellas zonas de la ciudad en las cuales se instrumentó la política (zc), y las zonas en donde no hay ubicada una preparatoria y, por lo tanto, donde no se instrumentó la política (ZNC), se hizo referencia en este trabajo al mismo objetivo geográfico político del radio de los tres kilómetros (véase cuadro 2). Es decir, se calcularon los niveles de marginación al interior de esos radios geográficos (zc), y luego se compararon con los niveles de marginación de las zonas fuera de esos radios geográficos (ZNC).

\section{Cuadro 2}

Definición de las zonas en comparación en este estudio

\begin{tabular}{ll}
\hline $\begin{array}{l}\text { Zonas con cobertura de preparatorias } \\
\text { de la Ciudad de México }\end{array}$ & $\begin{array}{c}\text { Zonas sin cobertura depreparatorias } \\
\text { dela Ciudad de México }\end{array}$ \\
\hline $\begin{array}{l}\text { El conjunto de las AGEB que se } \\
\text { encuentran en un radio de tres } \\
\text { kilómetros de donde está localizada } \\
\text { una preparatoria de la ciudad } \\
\text { de México }\end{array}$ & $\begin{array}{l}\text { El conjunto de las AGEB que no se } \\
\text { encuentran en el radio de tres } \\
\text { kilómetros de donde están } \\
\text { localizadas las preparatorias de la } \\
\text { ciudad de México }\end{array}$ \\
Número total de AGEB: 243 & Número total de AGEB: 1,666 \\
\hline
\end{tabular}

Fuente: Elaboración propia.

La unidad de información básica para construir la base de datos fue el Área Geoestadística Básica (АGEВ). Se agruparon las AGEB en las dos zonas de comparación: las zonas con cobertura ( $\mathrm{n}=243)$ y que están compuestas por los conjuntos de las AGEB que se hallan en un radio de tres kilómetros de distancia del plantel, y las zonas donde no hay una preparatoria $(n=1,666)$, es decir, las que están fuera de esos radios de cobertura de las preparatorias. ${ }^{4}$ A partir de la agregación de las AGEB en las dos zonas diferentes, se pudo hacer la comparación en los niveles de marginación con base en estadísticas descriptivas e inferenciales.

Una vez definidas las AGEB que integrarían cada zona -la de cobertura y la de no cobertura-, se procedió a calcular los Índices de Marginación (IM) con base en la definición y metodología del Consejo Nacional de Población (Conapo). Para Conapo, el IM es "una medida-resumen que permite diferenciar entidades fe-

\footnotetext{
${ }^{4}$ La Ciudad de México se halla divida en 1,909 AGEB.
} 
derativas y municipios según el impacto global de las carencias que padece la población, como resultado de la falta de acceso a la educación, la residencia en viviendas adecuadas, la percepción de ingresos monetarios insuficientes y las relacionadas con la residencia en localidad pequeñas" (Conapo, 2000: 11). Lo anterior deriva en una clasificación del grado de marginación de los estados y municipios de México, el cual puede ser: alto, medio alto, medio, bajo y muy bajo.

Los IM se calculan para los ámbitos estatal y municipal. No hay mediciones por debajo de ese nivel de agregación, por lo que procedimos por nuestra cuenta a calcular el IM para cada AGEB de la Ciudad de México, con lo que pudimos calcular el promedio para cada zona a compararse dentro de la ciudad (Zc versus ZNC).

Como mencionamos previamente, para el cálculo de los IM se siguió la misma metodología del Conapo, con la salvedad de que en este estudio no se pudieron incluir en el modelo dos variables socioeconómicas que el Conapo utiliza: el porcentaje de viviendas particulares con algún grado de hacinamiento, ${ }^{5}$ y el porcentaje de población en localidades con menos de cinco mil habitantes. ${ }^{6}$ En consecuencia, el IM utilizado en este estudio está compuesto por siete de las nueve variables del Conapo (véase cuadro 3). Es muy poco probable que los resultados de esta investigación vayan a variar considerando la naturaleza de las dos variables faltantes y la dirección de su relación con las demás.

Entonces, resumiendo, para poder calcular los IM en el nivel de AGEB para el Distrito Federal, en primer lugar se identificaron visualmente las AGEB que se hallaban en un radio de tres kilómetros a la redonda de cada uno de los planteles educativos del SBGDF por cada delegación. ${ }^{7}$ En segundo lugar, se tomó como base la metodología y las técnicas estadísticas utilizadas por el Conapo para la definición del IM, en el cual se consideran diferentes variables socioeconómicas que permiten identificar diversos rasgos o características de exclusión social. Como ya se dijo, puesto que el Conapo calcula los IM exclusivamente para los niveles estatal y municipal, fue requisito indispensable en este estudio cal-

\footnotetext{
${ }^{5}$ Debido a que no se cuenta con esta información para el nivel de AGEB.

${ }^{6}$ No se cuenta con esta información por AGEB, datos que de alguna manera pueden ser prescindibles debido a que este estudio se enfoca en un análisis urbano.

${ }^{7}$ En estos cálculos no se consideraron las tres delegaciones en las que no existe plantel, ni la delegación Magdalena Contreras, pues el plantel correspondiente funciona con una sede alterna en otra demarcación.
} 


\section{Cuadro 3}

Definición de las variables incorporadas en el cómputo del Índice de Marginación del Conapo y de este estudio

\begin{tabular}{|c|c|}
\hline $\begin{array}{l}\text { Variables que componen el Índice de } \\
\text { Marginación del Conapo: }\end{array}$ & $\begin{array}{l}\text { Variables que componen el Índice de } \\
\text { Marginación de este estudio: }\end{array}$ \\
\hline $\begin{array}{l}\text { 1. Porcentaje de población de } 15 \\
\text { años o más analfabeta }\end{array}$ & $\begin{array}{l}\text { 1. Porcentaje de población de } 15 \\
\text { años o más analfabeta }\end{array}$ \\
\hline $\begin{array}{l}\text { 2. Porcentaje de población de } 15 \\
\text { años o más sin primaria completa }\end{array}$ & $\begin{array}{l}\text { 2. Porcentaje de población de } 15 \\
\text { años o más sin primaria completa }\end{array}$ \\
\hline $\begin{array}{l}\text { 3. Porcentaje de ocupantes en vi- } \\
\text { viendas particulares sin agua en- } \\
\text { tubada }\end{array}$ & $\begin{array}{l}\text { 3. Porcentaje de ocupantes en vi- } \\
\text { viendas particulares sin agua en- } \\
\text { tubada }\end{array}$ \\
\hline $\begin{array}{l}\text { 4. Porcentaje de ocupantes en vi- } \\
\text { viendas particulares sin drenaje } \\
\text { ni servicio sanitario exclusivo }\end{array}$ & $\begin{array}{l}\text { 4. Porcentaje de ocupantes en vi- } \\
\text { viendas particulares sin drenaje } \\
\text { ni servicio sanitario exclusivo }\end{array}$ \\
\hline $\begin{array}{l}\text { 5. Porcentaje de ocupantes en vi- } \\
\text { viendas particulares con piso de } \\
\text { tierra }\end{array}$ & $\begin{array}{l}\text { 5. Porcentaje de ocupantes en vi- } \\
\text { viendas particulares con piso de } \\
\text { tierra }\end{array}$ \\
\hline $\begin{array}{l}\text { 6. Porcentaje de ocupantes en vi- } \\
\text { viendas particulares sin energía } \\
\text { eléctrica }\end{array}$ & $\begin{array}{l}\text { 6. Porcentaje de ocupantes en vi- } \\
\text { viendas particulares sin energía } \\
\text { eléctrica }\end{array}$ \\
\hline $\begin{array}{l}\text { 7. Porcentaje de población ocupa- } \\
\text { da con ingresos de hasta dos sala- } \\
\text { rios mínimos }\end{array}$ & $\begin{array}{l}\text { 7. Porcentaje de población ocupa- } \\
\text { da con ingresos de hasta dos sala- } \\
\text { rios mínimos }\end{array}$ \\
\hline \multicolumn{2}{|l|}{$\begin{array}{l}\text { 8. Porcentaje de viviendas con algún } \\
\text { porcentaje de hacinamiento }\end{array}$} \\
\hline \multicolumn{2}{|l|}{ 9. Porcentaje de población en loca- } \\
\hline $\begin{array}{l}\text { lidades con menos de } 5000 \text { habi- } \\
\text { tantes. }\end{array}$ & \\
\hline
\end{tabular}

Fuente: Elaboración propia con base en información del Conapo (2000).

cular el IM para cada AGEB por nuestra cuenta, asunto que explicamos en detalle a continuación.

Para calcular los IM, lo primero que se hizo fue conseguir los datos de las siete variables disponibles y estandarizar los valores de cada variable de la siguiente manera:

En donde:

$$
Z=\frac{x_{i}-\mu_{i j}}{s_{i j}}
$$

$\mathrm{Z}=$ es el valor estandarizado.

$x_{i}=$ es el indicador socioeconómico de la unidad de análisis. $\mu_{i}=$ es la media o promedio aritmético de los valores del indicador. 
$s_{i}=$ es la desviación estándar insesgada del indicador socioeconómico.

Una vez calculados los valores $\mathrm{z}-\mathrm{o}$ variables estandarizadas-, se realizó un análisis factorial con el procedimiento de componentes principales. Por medio del análisis factorial se logra transformar o factorizar un conjunto de variables en un factor o variable nueva. El objetivo es reelaborar una interpretación sencilla de un fenómeno original con base en un número menor de variables (Conapo, 2000). El análisis factorial es una de las herramientas estadísticas multivariantes más comunes para la reducción de datos en factores de información. Con este método se producen resultados compuestos que se pueden emplear en vez de utilizar un conjunto previo de una mayor cantidad de datos originales (Conrad, 1989).

\section{Cuadro 4}

\section{Pasos en el análisis estadístico}

1. Elaboración de la base de datos con las siete variables socioeconómicas disponibles para el nivel de AGEB.

2. Estandarización de las variables (valores Z).

3. Creación de los Índices de Marginación (IM) para cada AgeB por medio del análisis factorial.

4. Aplicación de la prueba Kolmogorov-Smirnov para probar la normalidad de los datos y, en consecuencia, elegir entre una técnica paramétrica versus una no paramétrica para la comparación de los promedios en los IM.

5. Vista la no-normalidad de los datos, siguió la aplicación de la prueba Mann-Whitney sobre diferencias en los promedios de los IM entre las áreas cubiertas por preparatorias de la Ciudad de México (zc) y las no cubiertas (ZNC).

Fuente: Elaboración propia.

Para obtener el primer componente estandarizado, el cual es una combinación lineal de las siete variables estandarizadas, se utilizó la siguiente fórmula: ${ }^{8}$

$$
Z_{i}=b_{1} x_{1}+b_{2} x_{2}+\ldots+b_{7} x_{7}
$$

En donde:

$\mathrm{Z}=$ es el factor o componente principal para cada unidad de datos i; o el índice de marginación para cada AGEB.

\footnotetext{
${ }^{8}$ Para la prueba se utilizó el paquete estadístico sPss versión 10.
} 
$\mathrm{b}=$ es el coeficiente de correlación para cada variable en relación con el factor.

$\mathrm{x}=$ es la variable incluida para el cálculo del factor

Una vez elaborados los IM para cada AGEB, se agregaron las AGEB de cada zona a compararse, se calcularon algunas estadísticas descriptivas, y se aplicó la prueba Kolmogorov-Smirnov para probar si los datos siguen una distribución normal o no; con base en ese procedimiento se puede seleccionar la técnica adecuada de comparación de promedios entre grupos. La lógica detrás de esta prueba es básicamente la de determinar si la distribución de una variable difiere significativamente de otra, en este caso de una curva normal.

Una vez visto que la distribución de los im en la Ciudad de México no sigue un comportamiento de curva normal, aplicamos la prueba Mann-Whitney para probar la hipótesis sobre diferencias en los rangos de los valores entre las áreas cubiertas y las no cubiertas por la política de preparatorias de la Ciudad de México.

La prueba Mann-Whitney es una técnica no paramétrica análoga a la prueba $t$ de Student (para dos muestras independientes), y se usa cuando la distribución de los valores no es normal, como es nuestro caso con los IM. Cabe mencionar que el valor del estadístico Z de Mann-Whitney sí se somete a una curva de probabilidades normal. La fórmula es la siguiente (procede en dos pasos):

Paso 1:

$$
U=n_{1} n_{2}+\frac{n_{1}\left(n_{1}+1\right)}{2}-R_{1}
$$

Paso 2:

$$
Z=\frac{U-\frac{n_{1} n_{2}}{2}}{\sqrt{\frac{n_{1} n_{2}}{n(n+1)}\left[\frac{\left(n^{3}-n\right)}{12}-\sum_{t}^{k} \frac{t_{i}^{3}-t_{i}}{12}\right]}}
$$


En donde:

$U=$ es el estadístico de Mann-Whitney.

$R=$ Suma de los rangos o rankings de un grupo.

$k=$ es el número de rangos distintos en los que hay empates.

$t=$ es el número de puntuaciones empatadas en cada rango.

$n=$ es el tamaño de cada muestra o grupo en comparación.

Para complementar nuestro análisis de localización, se hizo uso también de la información socioeconómica que contiene el Mapa mercadológico de la megaciudad de México de 1998 (Bimsa, 1998). En este mapa se hace una clasificación por delegación del ingreso familiar mensual y se establecen seis grupos en los que se divide el nivel de ingreso y de vida de los habitantes de la Ciudad de México (véase cuadro 5). En la definición de cada uno de estos parámetros se analizan además aspectos como: el perfil educativo, el perfil del hogar, el tipo de artículos que se poseen, los servicios que se utilizan, la diversión y el ingreso mensual. En este caso se realizó una inspección visual del mapa para conocer el nivel de ingreso y de vida de las zonas en donde están localizadas las preparatorias.

\section{Resultados}

Esta parte se divide en dos secciones: estadísticas descriptivas y pruebas de hipótesis. Al final hacemos un resumen de los hallazgos para pasar a la parte de la discusión y conclusiones.

\section{Cuadro 5}

Grupos de nivel de ingreso según el Mapa mercadológico de la megaciudad de México

1. $\mathrm{AB}+$ : Población con el más alto nivel de vida e ingresos en el país (más de 50 mil pesos).

2. C+: Población con ingreso y nivel de vida ligeramente superiores al medio (de 21 mil a 49 mil pesos).

3. C: Población con ingresos y nivel de vida medio (de 6 mil a 20 mil pesos).

4. D+: Población con ingresos y nivel de vida ligeramente por debajo del nivel medio (de 5 mil a 6 mil pesos).

5. D: Población con un nivel de vida austero y bajos ingresos (de 1,500 a 3 mil pesos).

6. E: Población con menores ingresos.

Fuente: Elaboración propia con base en información de Bimsa, 1998. 


\subsection{Estadísticas descriptivas}

Consideremos que al día de hoy existen 16 preparatorias funcionando, de las cuales 12 están ubicadas en sus sedes definitivas y otras cuatro se hallan ubicadas en espacios temporales. El análisis de este trabajo únicamente considera las ubicadas en sus sedes definitivas. ${ }^{9}$

Aunque originalmente se planteó establecer un plantel en cada una de las delegaciones políticas, las 16 escuelas están distribuidas en 13 delegaciones. En las delegaciones Benito Juárez, Cuauhtémoc y Venustiano Carranza no existen planteles, ya que de acuerdo con las autoridades del Instituto de Educación Media Superior del Distrito Federal (IEMS), en las dos primeras la oferta está completa y en la tercera no se ha podido encontrar un espacio. ${ }^{10}$ Las tres delegaciones que cuentan con dos planteles cada una son Tlalpan, Gustavo A. Madero e Iztapalapa (véase cuadro 6).

En el cuadro 7 se registra el número de personas con algún nivel de marginación en cada delegación. Las delegaciones Iztapalapa, Gustavo A. Madero y Álvaro Obregón son las que cuentan con el mayor número de personas en esta situación $(1,357,267$ personas, o $16 \%$ de la población total de la ciudad). Por otro lado, las delegaciones con el mayor número de personas sufriendo de muy altos niveles de marginación son Iztapalapa, Gustavo A. Madero y Xochimilco (en total, las tres delegaciones reportan 51,264 personas en esta situación, o $0.6 \%$ de la población total de la ciudad). En el lado contrario, las delegaciones con el menor número de personas con altos niveles de marginación son Benito Juárez, Miguel Hidalgo e Iztacalco. Nótese que las delegaciones con los menores IM son Benito Juárez, Coyoacán y Miguel Hidalgo.

En el cuadro 8 se muestra que la media de los IM en las zonas cubiertas por las preparatorias $(\mathrm{M}=-0.278)$ es menor frente al conjunto de las zonas no cubiertas $(\mathrm{M}=0.040)$. De acuerdo con la metodología del Conapo, cuanto más alejado de cero y en números negativos se encuentre el IM, mejor será el nivel de vida (o menor nivel de marginación, consecuentemente). Nótese lo cru-

${ }^{9}$ La razón por la que estos planteles no están aún en la sede definitiva se debe a que no se han podido encontrar los terrenos adecuados o a que las instalaciones están en remodelación. Datos obtenidos en entrevista con la Lic. Alicia Núñez, subdirectora Técnica del Instituto de Educación Media Superior del Distrito Federal.

${ }^{10}$ El listado con nombre y direcciones de los planteles se encuentra en: http:// www.iems.df.gob.mx/planteles/index.html 


\section{Cuadro 6}

Niveles de marginación y preparatorias del SBGDF en cada delegación de la Ciudad de México

\begin{tabular}{lcc}
\hline Delegación & $\begin{array}{c}\text { Lugar que ocupa en } \\
\text { el nivel de marginación }\end{array}$ & Número de preparatorias \\
\hline Milpa Alta & 1 & 1 \\
Tláhuac & 2 & 1 \\
Cuajimalpa & 3 & 1 \\
Xochimilco & 4 & 1 \\
Iztapalapa & 5 & 2 \\
M. Contreras & 6 & 1 \\
Álvaro Obregón & 7 & 1 \\
Tlalpan & 8 & 2 \\
Gustavo A. Madero & 9 & 2 \\
Iztacalco & 10 & 1 \\
Venustiano Carranza & 11 & 0 \\
Azcapotzalco & 12 & 1 \\
Miguel Hidalgo & 13 & 1 \\
Cuauhtémoc & 14 & 0 \\
Coyoacán & 15 & 0 \\
Benito Juárez & 16 & 1 \\
\hline
\end{tabular}

Fuente: Elaboración propia con base en datos del Conapo (2000) y del SBGDF (2002)

cial que esto resulta en nuestro análisis. Lo que esto significa es que, preliminarmente y en cuanto a estadística descriptiva, las preparatorias del SBGDF han sido localizadas en zonas de la ciudad donde, en promedio, las condiciones de vida son mejores que en el resto de la ciudad.

Con base en el Mapa mercadológico de la megaciudad de México, y en la revisión en el mismo de la ubicación de las preparatorias del SBGDF, se encontró que sólo uno de los planteles se encuentra situado en zonas de tipo E (Tlalpan I), seis de los centros educativos entran en el nivel D + (Tlalpan II, Tláhuac, Álvaro Obregón, Iztacalco, Milpa Alta y Azcapotzalco), tres en el nivel D (Gustavo A. Madero i y II, y la de Miguel Hidalgo), dos en el nivel C (Coyoacán y Xochimilco), y uno en el nivel AB (Cuajimalpa) (véanse los grupos de nivel de ingreso en el cuadro 5). Recordemos que son 16 preparatorias ubicadas en 13 delegaciones; de los 16 planteles, 12 ya están en su sede definitiva y cuatro en sedes temporales. Tres delegaciones no cuentan con planteles ni está contemplado que vayan a contar con alguno.

Por lo tanto, en segundo lugar, y también en cuanto a estadística descriptiva, podemos observar que el establecimiento de 15 de los 16 planteles (94\%) no corresponde con las zonas más 


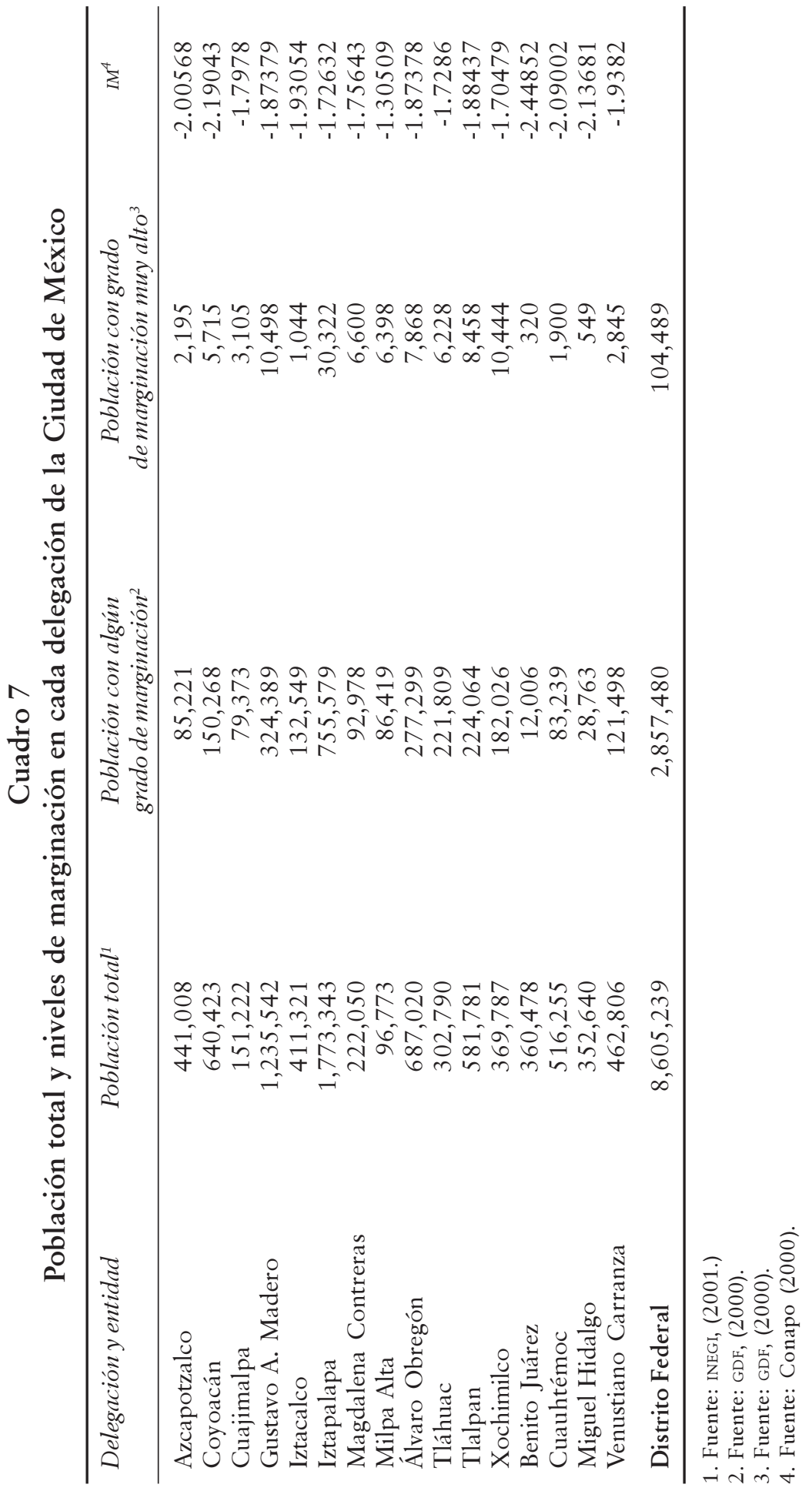




\section{Cuadro 8}

Estadísticas descriptivas sobre los índices de marginación por AGEB para la Ciudad de México y para cada zona

\begin{tabular}{lccc}
\hline Estadísticos & $\begin{array}{c}\text { Ciudad } \\
\text { deMéxico }\end{array}$ & $\begin{array}{c}\text { Zonas sincobertura } \\
\text { depreparatorias de } \\
\text { la Ciudad de México } \\
\text { (ZNC) }\end{array}$ & $\begin{array}{c}\text { Zonasconcobertura la } \\
\text { depreparatorias de } \\
\text { Cindad de México } \\
\text { (ZC) }\end{array}$ \\
\hline Media & 0.000 & 0.040 & -0.278 \\
Desviación estándar & 1.000 & 1.012 & 0.858 \\
N & 1,909 & 1,666 & 243 \\
Porcentaje & $100 \%$ & $87.2 \%$ & $12.8 \%$ \\
\hline
\end{tabular}

Fuente: Elaboración propia con base en datos del Conapo (2000).

marginadas; entendiéndose aquí mayor marginación como el nivel inferior de ingreso y de características habitacionales de la población en la zona. Según el Mapa mercadológico de la megaciudad de México, la mayoría de las preparatorias se encuentran en zonas donde el ingreso promedio se halla entre los tres mil y los cinco mil pesos mensuales. De acuerdo con estas consideraciones, sólo el plantel in de la delegación Tlalpan está ubicado en correspondencia con la población objetivo de la política; es un lugar en donde la población efectivamente cuenta con bajos estándares de calidad de vida.

\subsection{Pruebas de hipótesis}

En esta sección realizamos, en primer lugar, una prueba de normalidad Kolmogorov-Smirnov y encontramos que las distribuciones de valores de los IM en cada grupo de AGEB en las zonas cubiertas por preparatorias $(\mathrm{zC})$ versus las zonas no cubiertas por preparatorias (ZNC) no siguen un comportamiento normal. En el cuadro 9 se muestran los resultados de tal prueba y se muestra que ambas distribuciones tienen un comportamiento diferente a la curva normal $(\mathrm{z}=3.056, \mathrm{p}=.000) \cdot{ }^{11}$

Por lo tanto, fue requisito utilizar una técnica no paramétrica: de comparación entre grupos la prueba Mann-Whitney. Esta técnica procede tomando los valores de cada grupo -en este caso, de AGEB en las ZC y las ZNC- y transformándolos en rangos que posteriormente se comparan contra una media hipotética; o un rango promedio. Esta comparación/diferencia se somete a una curva de probabilidades normal.

${ }^{11}$ No se agregan los histogramas por brevedad de espacio. 


\section{Cuadro 9}

Resultados de la prueba Kolmogorov-Smirnov sobre la no-normalidad de las distribuciones de los IM de las zonas cubiertas (ZC) y las no cubiertas (ZNC) por una preparatoria de la Ciudad de México

\begin{tabular}{lcc}
\hline Estadístico & Valor & $\begin{array}{c}\text { Significancia estadistica } \\
\text { (prueba de dos colas) }\end{array}$ \\
\hline $\mathrm{z}$ de Kolmogorov-Smirnov $=$ & 3.056 & .000 \\
\hline
\end{tabular}

Fuente: Elaboración propia.

En el cuadro 10 se muestran los resultados de la prueba, los cuales sugieren tomar la hipótesis alternativa de una diferencia estadísticamente significativa entre los promedios de los niveles de marginación entre las dos áreas geográficas en cuestión $(\mathrm{z}=$ 6.032, $\mathrm{p}<.000)$.

\section{Cuadro 10}

Resultados de la prueba Mann-Whitney sobre diferencia en los rangos medios de los IM de las zonas cubiertas (zC) y las no cubiertas (ZNC) por una preparatoria de la Ciudad de México

\begin{tabular}{lcc}
\hline Estadístico & Valor & $\begin{array}{c}\text { Significancia estadística } \\
\text { (prueba de dos colas) }\end{array}$ \\
\hline $\mathrm{z}$ de Mann-Whitney $=$ & -6.032 & .000 \\
\hline
\end{tabular}

Fuente: Elaboración propia.

Recordemos que el promedio de los IM en cada zona era diferente, y para el caso de la zona sin cobertura de preparatorias del SBGDF (ZNC) ese promedio era mayor $(\mathrm{M}=0.040)$, y por lo tanto con mayores niveles de marginación (véase cuadro 7); este valor es más cercano a cero y en dirección a los números positivos de la escala.

En síntesis, los resultados de esta sección de estadística descriptiva y pruebas de hipótesis muestran que el promedio del IM de las zonas no cubiertas con planteles (ZNC) es mayor que en las zonas cubiertas (zc), y que esta diferencia difícilmente sería aleatoria $(\mathrm{p}=.000) .{ }^{12}$ Es decir, que la población que radica en las zonas que están cubiertas por una preparatoria del SBGDF posee,

\footnotetext{
${ }^{12}$ Realizamos la misma prueba con la técnica paramétrica $t$ de Student y los resultados fueron los mismos: rechazar Ho de que los promedios en las dos zonas son similares $(\mathrm{t}=5.282, \mathrm{p}=.000)$.
} 
en promedio, características socioeconómicas mejores que los de la población en las zonas no cubiertas por una preparatoria.

\section{Discusión y conclusiones}

Es claro que los beneficios sociales de impulsar la educación pública con la apertura de planteles son siempre perceptibles. El resultado de la política es meritorio por definición, muy especialmente en sociedades polarizadas en las cuales para los individuos, la educación es el único medio posible para mejorar sus condiciones de vida.

En este documento hemos realizado una evaluación de la lógica territorial de la política de educación media superior o del Sistema de Bachillerato del Gobierno del Distrito Federal; es decir, las preparatorias de la ciudad de México. Nos hemos enfocado en analizar el cumplimiento de uno de los objetivos geográficos de la instrumentación de esa política: la localización expresa de los planteles en las áreas más marginadas de la ciudad.

Los resultados del análisis indican que la ubicación geográfica de los planteles del SBGDF no corresponde con el objetivo geográfico predefinido. La evidencia y la serie de pruebas estadísticas realizadas en este trabajo nos permiten concluir que la localización no es la más deseable para efectos de una política redistributiva como es la política en cuestión. Es decir, los resultados muestran que los niveles promedio de marginación son incluso significativamente menores en las zonas atendidas o cubiertas por el conjunto de preparatorias (zc), que en las zonas de la ciudad no atendidas o descubiertas (ZNC), lo que nos permite inferir que la educación impartida en esos planteles no beneficia a la población más marginada de la ciudad.

Recordemos que otro de los objetivos geográficos de esa política era que en los planteles se atendería exclusivamente a los alumnos que vivieran en un radio de tres kilómetros a la redonda de cada plantel. Sin embargo, esta hipótesis geográfica no fue probada en este trabajo; queda pendiente su examen. Es decir, la pregunta se vuelve: ¿̇son realmente los residentes de las colonias en el radio de tres kilómetros los inscritos en esas preparatorias? Reafirmamos que esto es una pregunta cuya respuesta queda pendiente; nuestro análisis se limitó a la geografía de los planteles, no a la de los estudiantes.

Los resultados de esta investigación no son repetitivos ni confirmativos de análisis previos debido a la inexistencia de este tipo 
de estudios en la literatura especializada mexicana. Por lo mismo, los hallazgos son innovativos, de relevancia social, e invitan al análisis geográfico de otros tipos de servicios públicos. En general, abren la puerta a la prueba y comprensión estadística y geográfica de otras políticas urbanas.

Se concluye también que independientemente del incumplimiento del criterio geográfico, el curso de las acciones de la política del Gobierno del Distrito Federal sí logra tener un sentido redistributivo en su generalidad. La política es ineficaz en cuanto a su objetivo original de consumo colectivo de la población estudiante residente en las áreas más marginadas de la ciudad, pero no es, en lo absoluto, una política inútil para la ciudad. Esperamos que este tipo de estudios de evaluación política sea percibido positiva y constructivamente por la academia, el GDF y la sociedad civil, y que se traduzca en el mediano plazo en nuevas inversiones educativas mejor focalizadas y en nuevos y mejores estudios relacionados.

\section{Bibliografía}

Abrams, Philip (1978), "Towns and Economic Growth: Some Theories and Problems", en Philip Abrams, y Edward Wrigley, (eds.), Towns in Societies: Essays in Economic History and Historical Sociology, Cambridge University Press, Gran Bretaña, pp. 9-33.

Acosta, Adrián (2004), “Poder político, alternancia y desempeño institucional. La educación superior en Jalisco, 19952001”, Estudios Sociológicos, 22 (1): 53-78.

Barceinas, Fernando (2003), "Endogeneidad y rendimientos de la educación”, Estudios Económicos, 18 (1):79-131.

Bola, Ayeni (1979), Concepts and Techniques in Urban Analysis, St. Martin's Press, Nueva York.

Castells, Manuel (1974), La cuestión urbana, Siglo xxi, Editores, Madrid.

Conrad, Simon (1989), Assignments in Applied Statistics, John Wiley \& Sons Ltd, Gran Bretaña. 
Conapo (Consejo Nacional de Población) (2000), Indice de Marginación Nacional, Conapo, México, http://www.conapo. gob.mx

Esteve, Albert (2005), “Tendencias en homogamia educacional en México: 1970-2000”, Estudios Demográficos y Urbanos, 20 (2):341-362.

Garza, Gustavo y Jaime Sobrino (1989), Industrialización periférica en el sistema de ciudades de Sinaloa, El Colegio de México, México.

GDF (Gobierno del Distrito Federal) (2000), Breviarios Delegacionales, Dirección de Política Poblacional, GDF, México.

Handlin, Oscar (1967), "The Modern City as a Field of Historical Study”, en Oscar Handlin y John Burchard (eds.), The Historian and the City, The MIT Press, Cambridge, Mass., pp. 1-26.

Henderson, William y Larry Ledebur (1972), Urban Economics: Processes and Problems, John Wiley \& Sons, Nueva York.

Hirsch, Werner (1973), Urban Economic Analysis, McGraw-Hill, Nueva York.

INEGI (Instituto Nacional de Estadística, Geografía e Informática) (2007), Censo General de Población y Vivienda, Tabulados Básicos, Aguascalientes.

Bimsa (Buró de Investigación de Mercados) (1998), Mapa mercadológico de la megaciudad de México, XI edición, México.

Parker, Susan y Carla Pederzini (2000), “Género y educación en México”, Estudios Demográficos y Urbanos, 15 (1): 97-122.

Peterson, Paul (1981), City Limits, University of Chicago Press, Chicago.

Potters, Jan y Frans Van-Winden (1996), “Comparative Statics of a Signaling Game: An Experimental Study", International Journal of Game Theory, 25 (3):329-353. 
Sandler, Todd (1992), Collective Action: Theory and Applicatio$n s$, University of Michigan Press, Ann Arbor.

SBGDF (Sistema de Bachillerato del Gobierno del Distrito Federal) (2002), Propuesta Educativa SBGDF. México: Talleres de Corporación Mexicana de Impresión, México.

Stahl, Konrad y Pravin Varaiya (1983), "Local Collective Goods: A Critical Re-examination of the Tiebout Model", en: Jacaves François. Thisse y Henry .G. Zoller (eds.), Locational Analysis of Public Facilities, North-Holland, Amsterdam.

Sullivan, Arthur (1990), Urban Economics, Richard D. Irwin, Homewood, Illinois.

Vilalta, Carlos (2003), “Perspectivas geográficas en la sociología urbana: la difusión espacial de las preferencias electorales y la importancia del contexto local", Estudios Demográficos y Urbanos, 18 (3): 147-177.

Werna, Edmundo (2001), Combating Urban Inequalities, MPG Books Ltd, Reino Unido.

Laura Cervantes-Salcido. Maestra en Administración Pública y Políticas Publicas por la Escuela de Graduados en Administración Pública (EGAP) y Licenciada en Relaciones Internacionales por el Tecnológico de Monterrey, Campus Ciudad de México. Actualmente se desempeña como Directora de la Carrera en Relaciones Internacionales en el Tecnológico de Monterrey, Campus Santa Fe. Asimismo, colaboró como asistente del decano de la escuela de Humanidades y Ciencias Sociales (DHCS) en el Tecnológico de Monterrey, Campus Ciudad de México. Ha impartido desde hace 3 años la materia de Sociedad y Desarrollo en México, en la cual, se revisan los aspectos más importantes que han permitido el progreso social, económico y político de México.

Carlos Javier Vilalta-y Perdomo. Doctor en Estudios Urbanos por la Universidad Estatal de Portland y Maestro en Estudios Urbanos por El Colegio de México. Es actualmente Decano de la División de Administración y Ciencias Sociales del Tecnológi- 
co de Monterrey, Campus Santa Fe. Es profesor de las materias de Métodos Cuantitativos de Investigación; Desarrollo Regional; Análisis Espacial y Sistemas de Información Geográfica; Investigación Cuantitativa, Argumentación y Redacción para Revistas Científicas; Estadística Espacial; e Investigación Social y Pruebas No-Paramétricas. Por su labor docente fue reconocido como mejor profesor de la Escuela de Graduados en Administración y Políticas Públicas en el periodo 2003-2004. Ha sido investigador invitado en la Universidad de Carolina del Norte en Chapel Hill, es árbitro para las revistas Problemas del Desarrollo y Political Geography, es árbitro para el Fondo de Cultura Económica (FCE) en la sección de Arquitectura y Urbanismo, y pertenece al Sistema Nacional de Investigadores (sNI, nivel I). 